\title{
Centroid Based 3D Localization Technique Using RSSI With a Mobile Robot
}

\author{
Amarlingam M, P Rajalakshmi \\ Department of Electrical Engineering \\ Indian Institute of Technology Hyderabad \\ Hyderabad, India \\ Email: ee13p1003, raji@iith.ac.in
}

\author{
Masaya Yoshida, Kiyohito Yoshihara \\ KDDI R\&D Laboratories Inc. \\ Green and M2M Application Laboratory \\ Iidabashi, Japan \\ Email: my-yoshida, yosshy@kddilabs.jp
}

\begin{abstract}
Knowledge of sensor node 3D location in a sensor network is more important, because many practical applications needs to know the location of sensor data source. This paper presents a new technique for finding indoor 3D location of a sensor node by using Received Signal Strength Indication (RSSI). Proposed localization algorithm is derived from centroid algorithm with composition of empirical path loss model. It gives less error in estimating 3D location of sensor node in a sensor network when compared to its actual location. Algorithm has been implemented and analysed by using IITH motes and a Mobile Robot.
\end{abstract}

Index Terms-RSSI, Centroid algorithm, 3D localization.

\section{INTRODUCTION}

Development in wireless communications and electronics has enhanced micro sensors technology, smartness in controlling and monitoring sensors in a sensor network [1]. Wireless sensor networks are used for sensing physical factors like temperature, humidity and also for monitoring and detecting environmental factors, chemicals, smoke etc. There are many theoretical and practical works for designing and deployment of wireless sensor network. Authors of [2] proposed a deployment tool for wireless sensor network. After deployment phase, knowledge of sensor node location is crucial in many practical applications like forest fires location detection, marine monitoring, animal monitoring and so on. For outdoor localization GPS is used, but in indoor environment GPS does not work. Most of the localization works are based on 2D localization. In [3] and [4] they discussed RSSI based localization algorithms for 2D. In real-time applications, sensor nodes will be placed in three-dimensional space. Our work is concerned with mobile Robot based 3D localization of a sensor node in a sensor network. We use Roomba machine, termed as mobile Robot as shown in Fig.1.

Paper [5] provides survey on 3D localization of sensor nodes. Existing algorithms on localization can be divided into two types as described in [9]. One is co-ordinate based localization and other is co-ordinate free localization (Fig.2). Co-ordinate based localization algorithms depends on RSSI and path loss modelling of environment as discussed in [6]. We focused on co-ordinate based localization algorithm. In most of the 2D and 3D localization techniques anchor nodes are used to collect RSSI values as discussed in [7]. Estimated

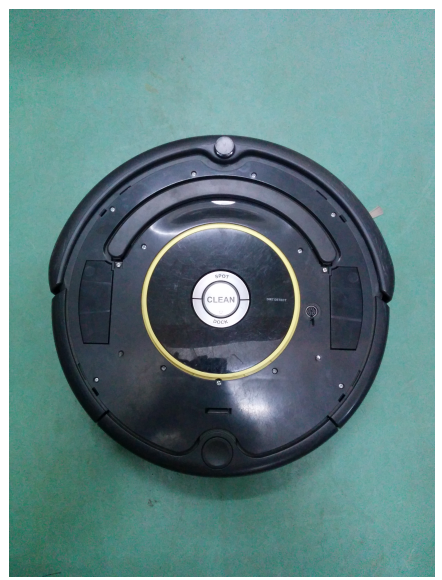

Fig. 1: Roomba machine-Mobile Robot

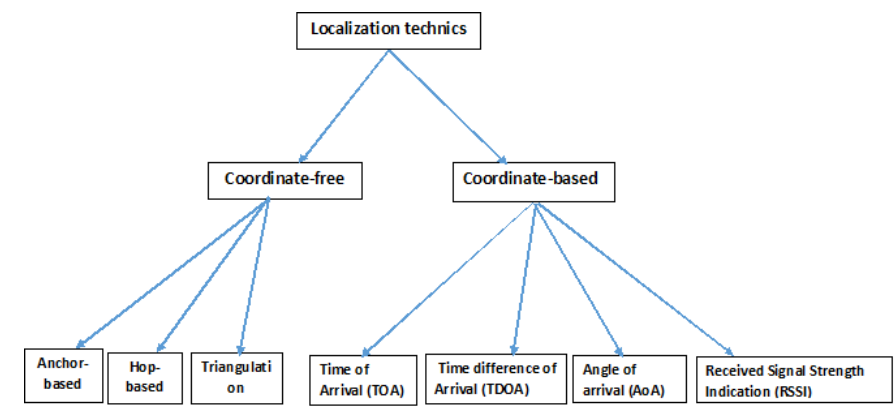

Fig. 2: Classification of localization algorithms

location accuracy depends on number of anchor nodes used. System cost will increase with increasing of anchor nodes. To eliminate this problem we have used moving Robot (Roomba) as the anchor node. Roomba provides user interface to program it easily. We used Roomba instead of using more number of anchor nodes. Here we place one sensor node on Roomba, called as beacon node. Roomba moves in defined area to estimate location of a target node. Roomba will have its location details. The Roomba location will be the location of beacon node as it is placed on Roomba and also beacon node will have RSSI values with respect to target node. Our algorithm is composition of basic centroid finding method and RSSI based location finding method. For finding distance 


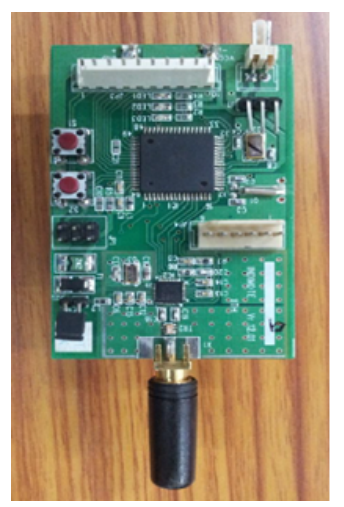

Fig. 3: IITH Mote

between two nodes, we use path loss model. In [8] and [9] authors discussed about basic centroid method considering RSSI values. Authors of [10] proposed a practical path loss model for indoor localization.

In this paper we propose a 3D localization algorithm, which is less cost and will give less error in predicting a target node location. Proposed 3D localization algorithm has been tested using an in house developed IITH Mote shown in Fig.3, which makes use of IEEE 802.15.4 standard for PHY and MAC layers [14].

The rest of the paper is organized as follows. Section II describes proposed 3D localization algorithm. Section III describes the experimental setup. Section IV describes experimental results and analysis. Finally, section V concludes the paper, with future scope of work.

\section{Proposed 3D LOCALIZATION ALGORITHM}

Centroid Location (CL) algorithm is well known for finding target node positions [12]. In CL algorithm, beacon nodes initially knows their location information, then broadcasts their position details to all the remaining nodes which are in network range. After the target node gets all beacon nodes information, it calculates position $T_{e}(x, y)$ from $\mathrm{n}$ beacon nodes as shown in equation (1). The localization error $\mathrm{E}(\mathrm{x}, \mathrm{y})$ is defined as distance between estimated target position $\left(T_{e}(x, y)\right)$ to actual target position $(T(x, y))$ as shown in equation (2).

$$
\begin{gathered}
T_{e}(x, y)=\frac{\sum_{j=1}^{n} B_{j}\left(x_{j}, y_{j}\right)}{n} \\
B_{j}\left(x_{j}, y_{j}\right) \rightarrow j^{t h} \text { beacon position. } \\
E(x, y)=\left|T(x, y)-T_{e}(x, y)\right|
\end{gathered}
$$

In this paper, it is assumed that target node is placed above the ground level within considered experimental area. Mobile Robot moves on the ground within the experimental area and passes below the target node. In our proposed 3D localization algorithm, the defined area is divided into grids

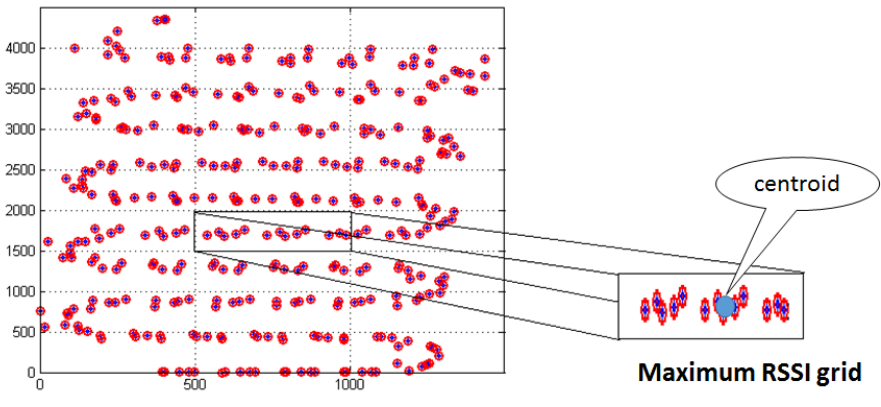

Fig. 4: Max. RSSI value grid and centroid finding

and average RSSI value is computed over each grid. The grid with maximum average RSSI value is considered, then the centroid $\left(X_{e}, Y_{e}\right)$ of the grid is calculated using equation (1) as shown in Fig.4. After finding centroid in 2D plane, to estimate height of the target node we need distance between target node and beacon positions which are in maximum averaged RSSI value grid. In order to estimate distances between beacon positions and target node path loss model is used. Here we estimate distances between each beacon node position to target node by using RSSI value of known beacon positions. In our algorithm, Revised Hata Okumara model [11] is used to calculate distance from RSSI values of beacon positions for indoor localization as shown in equation (3).

$$
\begin{array}{r}
\log D_{e i}=\frac{1}{10 \eta}\left[P_{T X}-P_{R X i}+G_{T X}+G_{R X}-X_{\alpha}+\right. \\
20 \log \lambda-20 \log (4 \pi)]
\end{array}
$$

Where $D_{e i} \rightarrow$ estimated distance between the target node

$$
\left(X_{e}, Y_{e}, Z_{e}\right) \text { and } i^{\text {th }} \text { Beacon node }\left(X_{i}, Y_{i}\right) \text {. }
$$

$G_{T X}(d B i) \rightarrow$ Transmit antenna gain

$G_{R X}(d B i) \rightarrow$ Receiver antenna gain

$P_{T X}(d B m) \rightarrow$ Target node transmit power

$P_{R X i}(\mathrm{dBm}) \rightarrow$ Measured received power at $i^{t h}$ beacon position

$\eta \rightarrow$ Measure of influence of obstacle like partitions and obstacles in indoor environment ranges from 4 to 5 and for free space it is equal to 2 .

$X_{\alpha} \rightarrow$ Normal random variable with standard deviation of $\alpha$ and varies from $3 \mathrm{~dB}$ to $20 \mathrm{~dB}$.

$P_{R X i}(d B m)$, measure received power at beacon positions from respective RSSI values is given by equation (4). This is specified in IITH Mote radio IC [13].

$$
P_{R X i}=-91+3\left(R_{i}-1\right)
$$

Where $R_{i} \rightarrow$ RSSI value of $i^{t h}$ beacon position. 


\begin{tabular}{|l|l|}
\hline Parameter & Value \\
\hline$P_{T X}$ & $-17.2(\mathrm{dBm})$ \\
\hline$G_{T X}$ & $0(\mathrm{dBi})$ \\
\hline$G_{R X}$ & $0(\mathrm{dBi})$ \\
\hline$X_{\alpha}$ & $3(\mathrm{~dB})$ \\
\hline$\lambda$ & 0.1250 (meters) \\
\hline$\eta$ & 2 \\
\hline
\end{tabular}

TABLE I: Path loss model parameter values used

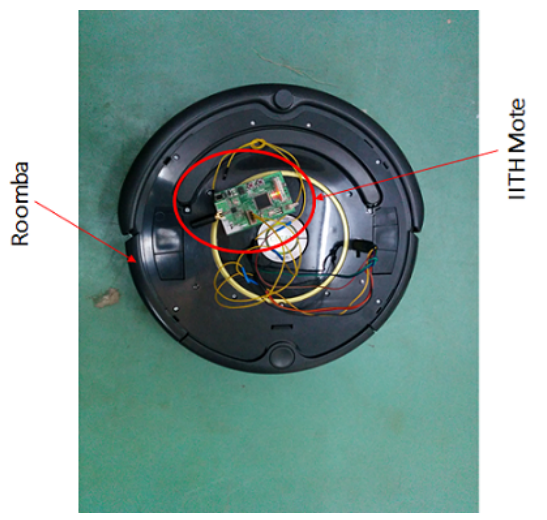

Fig. 5: IITH Mote interfacing with Roomba

TABLE.I shows path loss model parameters considered for experimentation. To estimate height of target node i.e $\mathrm{Z}$ axis value, we used Euclidian distance formula (5).

$$
D_{e i}=\sqrt{\left(X_{i}-X_{e}\right)^{2}+\left(Y_{i}-Y_{e}\right)^{2}+\left(Z_{e i}\right)^{2}}
$$

Where $D_{e i}$ can be computed from path loss model (3), $\left(X_{e}, Y_{e}\right)$ values from Centroid location algorithm (1). Maximum range expected between beacon positions and target node is $5.95 \mathrm{~m}$, calculated from experimental area and height of the room.

$\left(X_{i}, Y_{i}\right)$ values are beacon positions of maximum average RSSI value grid. Substituting these values in (5) gives unknown values $Z_{e i}$, which is estimated $\mathrm{Z}$-axis value of target node with respect to $i^{\text {th }}$ beacon position. From all $\mathrm{n}$ beacon positions we get n size vector of $Z_{e i}$. To estimate $Z_{e}$, we find mean of it as given in (6).

$$
Z_{e}=\frac{\sum_{i=1}^{n} Z_{e i}}{n}
$$

The pseudo code for proposed 3D localization algorithm is described in Algorithm 1.

\section{EXPERIMENTAL SETUP FOR 3D LOCALIZATION USING ROOMBA}

The experiment is performed in a room by considering area $1700 \mathrm{~mm} * 4500 \mathrm{~mm}$. Height of the room is $3500 \mathrm{~mm}$ and the area is divided into grids size of $0.5 \mathrm{~m}^{*} 0.5 \mathrm{~m}$. Considered experimental area is empty and remaining area of the room is having furniture. We took a moving Robot Roomba [15] and fixed one sensor node (Beacon node IITH Mote) as shown in Fig.5. The beacon node is programmed to give instruction
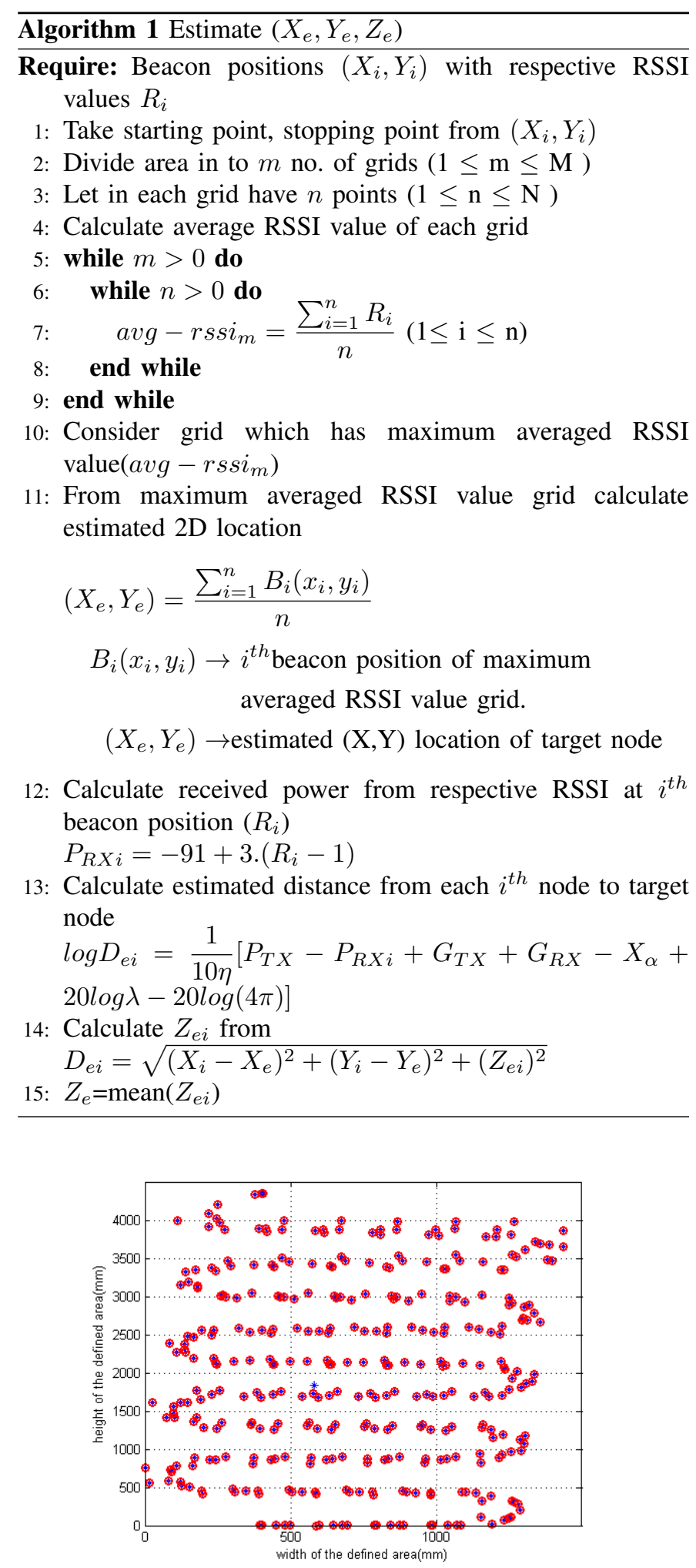

Fig. 6: Roomba moved path in defined area from top view

of movement to Roomba through serial port communication. Beacon node sends command to Roomba to move in zigzag way in a defined area as shown in Fig.6. Target node is placed in defined 3D location and it is also programmed to send 


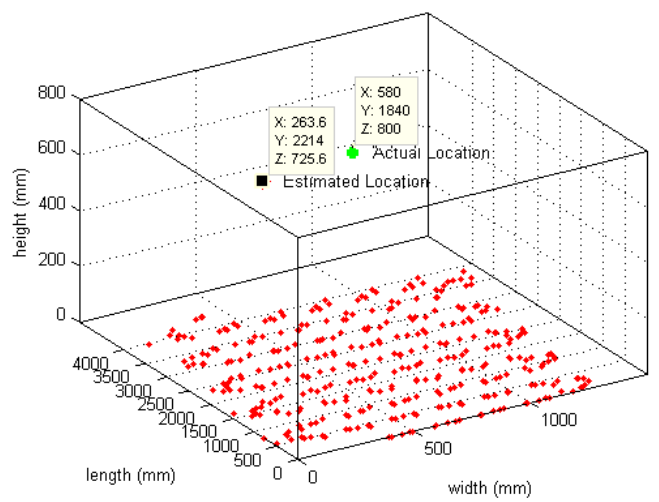

Fig. 7: Estimated position and actual position at height $=$ $800 \mathrm{~mm}$

periodical dummy packets through wireless communication to beacon node with a period of 250 milliseconds. While Roomba moving in zigzag way in the defined area, beacon node get packets periodically from target node. Beacon node calculates RSSI values and has its position details received from Roomba (by sending commands through serial communication to Roomba) with respect to Roomba movement. Zigbee 802.15 .4 with frequency of $2.4 \mathrm{GHz}$ is used for wireless communication and to get RSSI values. After getting RSSI values and respective position details from Roomba it will send a Location packet (a packet consists RSSI values and respective position details) through wireless communication to base node which is connected to Server (Server is a PC which takes data from base node through serial port). Base node is a sensor node which is programmed to receive data from beacon node and send it to Server. At Server our algorithm runs to estimate 3D location of target node. For the communication between base node and Server through serial port we used java program. After getting results of RSSI values with respective position details we used matlab to analyse data and to apply our algorithm. For programming beacon node, target node, base node we used Tinyos-2.1.1 nesC Script. In brief all software's used for experimental setup are tabulated in TABLE.II.

\begin{tabular}{|l|l|}
\hline nesC & Program to IITH Motes \\
\hline Matlab & proposed algorithm implemented at the Server \\
\hline Java & Data collection at Server \\
\hline
\end{tabular}

TABLE II: Programming languages used

\section{EXPERIMENTAL RESULTS ANALYSIS}

Experiment is performed by placing target node at three different heights. Actual and estimated positions for this three different heights are shown in Fig.7, Fig.8 and Fig.9. Let us consider the scenario of 3D localization as shown in Fig.7 for further analysis. From Fig.7 $11^{\text {th }}$ grid has maximum average

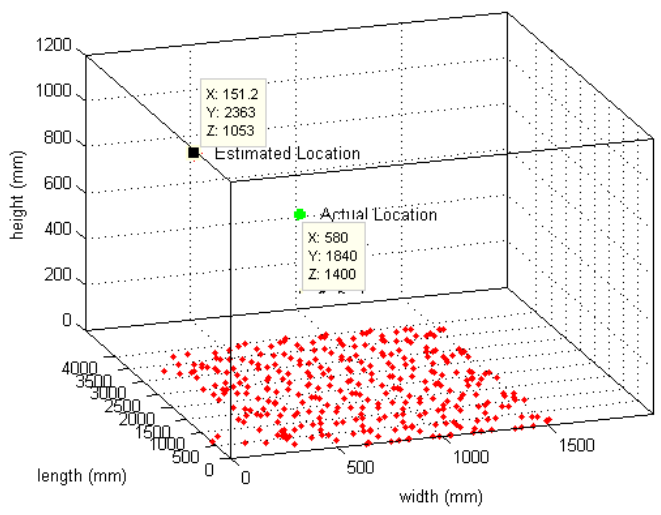

Fig. 8: Estimated position and actual position at height $=$ $1400 \mathrm{~mm}$

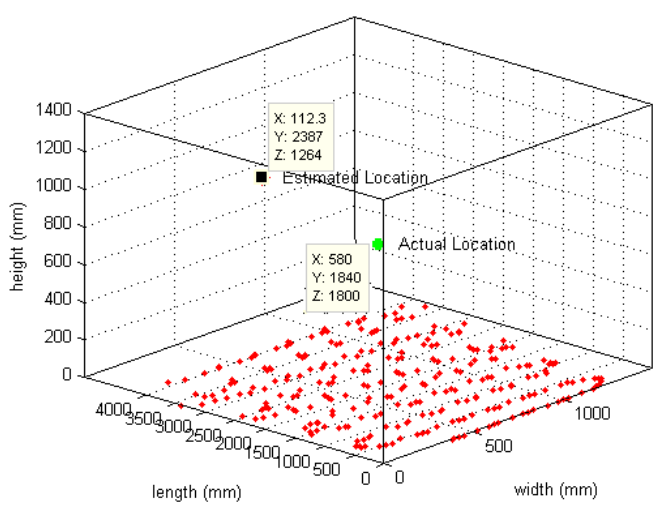

Fig. 9: Estimated position and actual position at height $=$ $1800 \mathrm{~mm}$

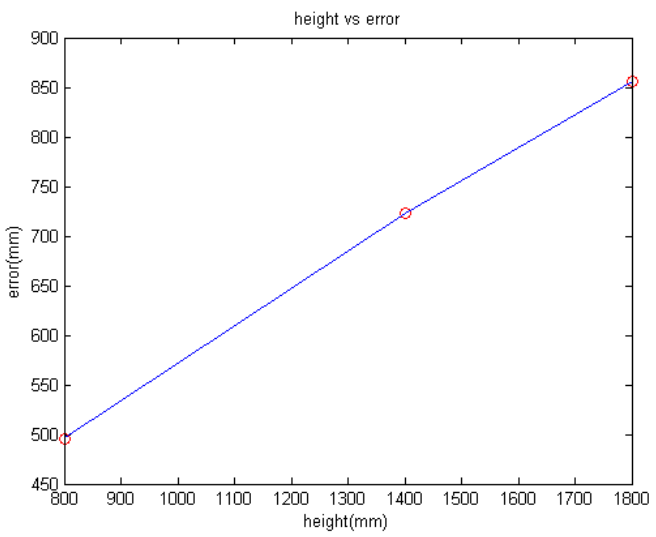

Fig. 10: Predicted Location error

RSSI value of $-66.0750 \mathrm{dBm}$. This grid consists 13 beacon locations $\left(X_{i}, Y_{i}\right)$, and calculated centroid $\left(X_{e}, Y_{e}\right)$ is (263.6, 2214)mm by using equation (1). From 13 beacon positions $\left(X_{i}, Y_{i}\right)$ and respective RSSI values $R_{i}$, distance vector $D_{e i}$ of size $1 \times 13$ is calculated by using equation (3). $Z_{e i}(1 \times 13)$ is obtained by substituting $\left(X_{i}, Y_{i}\right),\left(X_{e}, Y_{e}\right)$ and $D_{e i}$ values 
in equation (5). Finally $Z_{e}$ calculated from equation (6) using $Z_{e i}$ vector of size $1 \times 13$ and $Z_{e}$ value is $725.6 \mathrm{~mm}$. Locations of estimated and actual target node positions and error is tabulated in TABLE.III. Error versus height of the target node is plotted in Fig.10. Experimentation results show that proposed algorithm can estimate target node location with less than 1 meter estimation error.

\begin{tabular}{|l|l|l|}
\hline Actual location $(\mathrm{X}, \mathrm{Y}, \mathrm{Z})$ & Estimated location $\left(X_{e}, Y_{e}, Z_{e}\right)$ & Error \\
\hline$(580,1840,800) \mathrm{mm}$ & $(263.6,2214,725.6) \mathrm{mm}$ & $495.7 \mathrm{~mm}$ \\
\hline$(580,1840,1400) \mathrm{mm}$ & $(151.2363,2363.5,1053.1) \mathrm{mm}$ & $722.4 \mathrm{~mm}$ \\
\hline$(580,1840,1800) \mathrm{mm}$ & $(112.2869,2386.5,1263.7) \mathrm{mm}$ & $855.8 \mathrm{~mm}$ \\
\hline
\end{tabular}

TABLE III: Error comparison

\section{CONClusion}

In this paper we proposed a 3D localization algorithm for knowing the location of sensor node in a sensor network. We used IITH Mote and mobile Robot to perform experiment in real field deployment area (Indoor). The experimental results shows that the proposed algorithm gives very less error of 3D location of target node ( $<1$ meter). Our future plan is to develop a dynamic algorithm to get still more accurate $3 \mathrm{D}$ location of a desired sensor node.

\section{ACKNOWLEDGMENT}

We would like to thank Mr. Hanumantha Rao Madala for his support in Server programming part of the project.

\section{REFERENCES}

[1] Seung-Ho Baeg, Jae-Han Park, Jaehan Koh, Kyung-Wook Park, Moon-Hong Baeg. "RoboMaidHome: A Sensor Network-based Smart Home Environment for Service Robots", 16th IEEE International Conference on Robot \& Human Interactive Communication 26-29, Augus 2007 / Jeju, Korea.

[2] Amarlingam M, Adityan I, P Rajalakshmi, Yasutaka Nishimura, Masaya Yoshida, Kiyohito Yoshihara. "Deployment Adviser tool for Wireless Sensor Networks", IEEE World Forum on Internet of Things 2014.

[3] Masashi Sugano, Tomonori Kawazoe, Yoshikazu Ohta, and Masayuki Murata. "Indoor Localization System using RSSI measurement of Wireless Sensor Network based on Zigbee standard", Wireless Sensor Networks 2006.

[4] Gang Wang, Kehu Yang. "A New Approach to Sensor Node Localization using RSS Measurements in Wireless Sensor Networks", IEEE TRANSACTION ON WIRELESS COMMUNICATIONS, VOL. 10,NO. 5,MAY 2011.

[5] Shayon Samanta, Prof. Punesh, U.Tembhare, Prof. Charan, R. Pote. "A Survey on 3D Localization in Wireless Sensor Networks", International Journal Of Computational Engineering Research Vol.3 Issue. 1 JANUARY 2013.

[6] Sabastin Gansemer, Uwe GroBmann, Syuzanna Hakobyan."RSSI-based Euclidean Distance Algorithm for Indoor Positioning adapted for the use in dynamically changing WLAN environments and multi-level buildings ", International Conference on Indoor positioning and Indoor Navigation(IPIN) 2010.

[7] Xiufang FENG, Zhongyi XIAO, Xiufeng CUI. "Improved RSSI Algorithm for Wireless Sensor Networks in 3D", Journal of Computational Information System 7: 16(2011)5866-5873.

[8] John Blumenthal, Ralf Grossmann, Frank Golatowaski, Dirk Timmermann. "Weighted Centroid Localization in Zigbee-based Sensor Networks", Intelligent Signal Processing, 2007. WISP 2007.

[9] Stephan Schuhmann, Klaus Herrmann, Kurt Rothermel, Jan Blumenthal, Dirk Timmermann. "Improved Weighted Centroid Localization in Smart Ubiquitous Environments", Ubiquitous Intelligence and Computing Lecture Notes in Computer Science Volume 5061,2008,pp 20-34.

[10] Mardeni R, Solahuddin Y."Path Loss Model Development for Indoor Signal Loss Prediction at $2.4 \mathrm{GHz}$ 802.11n Network", Internation conference on Microwave and Millimeter Wave Techmnology(ICMMT) 2012.

[11] Atreyi Bose and Chua Heng Foh."A Practical Path Loss Model for Indoor Wifi Positioning Enhancement",ICICS 2012

[12] N. Bulusu, J. Heidemann, and D. Estrin, "GPS-less low cost outdoor localization for very small devices",IEEE Personal communications Magazine, 7(5):28-34,October 2000.

[13] Atmel, "Low Power $2.4 \mathrm{GHz}$ Transceiver for ZigBee, IEEE 802.15.4, 6LOWPAN, RF4CE and ISM Applications", 5131E-MCU Wireless Feb 2009.

[14] http://www.iith.ac.in/ raji/downloads/IITH-motewebpage.pdf

[15] http://www.irobot.com 